\title{
Editing genomic DNA in cancer cells with high genetic variance: Benefit or risk?
}

\author{
LIN WANG $^{1}$, YIXIANG WANG ${ }^{2}$ and CHUANBIN GUO ${ }^{1}$ \\ ${ }^{1}$ Department of Oral and Maxillofacial Surgery; ${ }^{2}$ Central Laboratory, Peking University School \\ and Hospital of Stomatology, Beijing 100081, P.R. China
}

Received December 21, 2013; Accepted February 7, 2014

DOI: 10.3892/or.2014.3067

\begin{abstract}
The generation of stably-transfected cell lines is a common and very important technology in cancer science. Considerable knowledge in the field of life sciences has been gained through the modification of the genetic code. However, there is a risk in evaluating exogenous gene function through editing genomic DNA in a cancer cell with high genetic variance. In the present study, we showed that genomic DNA status should be considered when evaluating the exogenous gene function in a cancer cell line with high variant genome through stable transfection technology, immunostaining, wound healing assay, Transwell invasion assay, real-time PCR, western blot and karyotyping analysis. Our results showed that the S100P expression level was not related to the migration and invasion abilities in these stably transfected cell lines derived from a human salivary adenoid cystic carcinoma cell line SACC-83. The MMP expression pattern was detected by western blot analysis which matched the biological behaviors in these cells. The genomic analysis showed that SACC-83 presented hypotetraploid karyotyping with high variance. Our data indicated that establishment of stable transgenic cancer cell lines should consider the status of genetic variance in a cancer cell to avoid any biased conclusion.
\end{abstract}

\section{Introduction}

The generation of stably-transfected cell lines is a common and very important technology in the life sciences (1). It is

Correspondence to: Professor Chuanbin Guo, Department of Oral and Maxillofacial Surgery, Peking University School and Hospital of Stomatology, 22 Zhongguancun Avenue South, Haidian, Beijing 100081, P.R. China

E-mail:guodazuo@sina.com

Professor Yixiang Wang, Central Laboratory, Peking University School and Hospital of Stomatology, 22 Zhongguancun Avenue South, Haidian, Beijing 100081, P.R. China

E-mail: kqwangyx@bjmu.edu.cn

Key words: genome, genetic variation, transfection, gene transfer techniques commonly practiced in order to understand a specific gene's function in the cells or organisms (2). In contrast to transient expression, stable expression allows long-term and defined investigation of the gene of interest (3). To date, considerable achievements have been made using this technology (4-6); researchers in the life sciences have gained significant insight through the modification of the genetic code.

It is well known that phenotypic changes are a result of multiple genetic interactions and are often complex. Exogenous DNA could be inserted into genome through transfection technology and exert its function after it is introduced into a cell under selection stress, to finally generate the stable modified cell lines with the desired characteristics (7). However, the technique has its limitation due to the randomness of incorporation of exogenous gene and the possibility of affecting other gene expression patterns (1), especially for introducing exogenous DNA into cancer cells that already have high genetic variance (8). Although the desired expression pattern is obtained in the stably transformed cancer cell line, the characteristics of the stable cell line may not be in line with the function of exogenous DNA.

In the present study, we provided evidence of the effect of exogenous DNA stress on the required function of the transformed cells. Overexpression plasmid containing exogenous S100P gene (S100P) and knockdown plasmid containing shRNA targeted to S100P sequence (shS100P) were transfected into a human salivary adenoid cystic carcinoma (SACC) cell line SACC-83. Then, the cell mobility and invasion ability were detected and the results showed that the characteristics of these SACC-83-derived stably transfected cell lines were not governed by S100P expression. Therefore, we suspected that genomic instability caused by exogenous DNA stress could determine the required characteristics in SACC- 83 based cell lines.

\section{Materials and methods}

Construction of S100P overexpression plasmid and S100P knockdown plasmid. Human S100P gene was generated from SACC-LM cDNA by reverse transcription-polymerase chain reaction using the following primers: forward primer containing EcoRI restriction endonuclease site in the 5' end (5'-TTGAATTCATGACGGAACTAGAGACAGCCATG-3') and reverse primer with $B a m H I$ restriction endonuclease site 
in the 5 ' end (5'-TTGGATCCTCATTTGAGTCCTGCCTT CTCAAAG-3'). Restriction enzyme sites are underlined. The PCR fragment was cut by EcoRI and BamHI, and then subcloned into pCDNA3.1 vector to generate an expression construct, pCDNA-S100P. The identity of the coding sequence for S100P in the pCDNA vector was confirmed by DNA sequencing. The S100P gene-specific shRNA expression pRI-GFP/Neo-shS100P plasmid was constructed using synthetic oligonucleotides cloned into pRI-GFP/Neo plasmid. The sequence AATGGAGATGCCC AGGTGGAC is designed for specific targeting of human S100P gene.

Cell cultures and establishment of stable cell lines. Human SACC cell line SACC-83 was used for the establishment of stably transfected SACC-83-derived cell lines through transfection of pCDNA-S100P, pRI-GFP/Neo-shS100P, pCDNA3.1 plasmids, respectively. Briefly, S100P overexpression and S100P knockdown plasmids were respectively transfected into SACC-83 with the Lipofectamine 2000 according to the manufacturer's instructions (Invitrogen, Carlsbad, CA, USA). Stable cell lines derived from single colony were established following selection with $1,000 \mu \mathrm{g} / \mathrm{ml} \mathrm{G} 418$ (Sigma-Aldrich, St. Louis, MO, USA), then evaluated by testing S100P expression via real-time PCR and western blot analysis. Since empty vectors pCDNA3.1 and pRI-GFP/Neo generated stably transfected cell lines had the similar characteristics of migration and invasion (data not shown), hereafter, only the pCDNA3.1 stably transfected cell line, named SACC-83-MOCK was used as the mock control. SACC-83 and SACC-83-derived cell lines (SACC-83-S100P, SACC-83-shS100P and SACC-83-MOCK) were cultured in RPMI-1640 (Gibco, Grand Island, NY, USA) supplemented with $10 \%$ fetal bovine serum (FBS; Thermo Fisher Scientific Inc., Waltham, MA, USA), $100 \mathrm{U} / \mathrm{ml}$ penicillin and $100 \mu \mathrm{g} / \mathrm{ml}$ streptomycin (Gibco) and maintained in a humidified incubator at $37^{\circ} \mathrm{C}$ with $5 \% \mathrm{CO}_{2}$ for the following experiments.

Immunocytochemical staining. The S100P protein was detected using a labeled streptavidin-biotin method after antigen retrieval. The cells were cultured on the glass coverslips at $37^{\circ} \mathrm{C}$ in a humidified $\mathrm{CO}_{2}$ incubator until they were $50-70 \%$ confluent. They were then fixed with $4 \%$ paraformaldehyde for $10 \mathrm{~min}$ and subjected to immunocytochemistry. The glass coverlids were rinsed twice with PBS, and endogenous peroxidase was blocked by the use of 3\% hydrogen peroxide for $10 \mathrm{~min}$. The samples were blocked with normal goat serum for $30 \mathrm{~min}$, incubated with anti-human anti-S100P polyclonal antibody (Epitomics, Burlingame, CA, USA) overnight at $4^{\circ} \mathrm{C}$, followed by peroxidase-conjugated immunoglobulin for $30 \mathrm{~min}$, developed for color with peroxidase substrate 3,3'-diaminobenzidine (DAB), counterstained with hematoxylin, and recorded using an Olympus DP Controller.

Quantitative PCR ( $q P C R)$ analysis. Total RNA was extracted from SACC-83 or its derived cell lines using the TRIzol reagent according to the manufacturer's instructions (Invitrogen). Complementary DNA was reverse transcribed by the use of $2.5 \mu \mathrm{g}$ of RNA as template. qPCR was performed using the ABI 7500 Real-Time PCR machine (Applied Biosystems, Carlsbad, CA, USA) coupled with SYBR-Green chemistry (Roche Diagnostics, Indianapolis, IN, USA). All PCR reactions were in $20 \mu \mathrm{l}$ of total volume containing $10 \mu \mathrm{l}$ of SYBR-Green PCR Master Mix, $50 \mathrm{ng}$ cDNA, $200 \mathrm{nM}$ of the following primer sets: S100P (5'-ATGACGGAACTAGAGAC AGCC-3' and 5'-AGGAAGCCTGGTAGCTCCTT-3'). The housekeeping gene glyceraldehyde-3-phosphate dehydrogenase (GAPDH) was used as an internal control (5'-ATGGGG AAGGTGAAGGTCG-3' and 3'-GGGGTCATTGATGGCAA CAATA-5'). All amplifications were carried out in triplicate for each sample and repeated three times. The thermal cycling was $10 \mathrm{~min}$ at $95^{\circ} \mathrm{C}$, followed by 40 cycles at $95^{\circ} \mathrm{C}$ for $15 \mathrm{sec}$, at $60^{\circ} \mathrm{C}$ for $60 \mathrm{sec}$. The specificity of amplification was monitored using the dissociation curve of the amplified products. Relative expression of the target genes was calculated using the $2^{-\Delta \Delta C t}$ method.

Western blot analysis. Cells were harvested and lysed in RIPA buffer with protease inhibitors (Roche Diagnostics). Protein concentration was determined using the BCA Protein Assay (Thermo Fisher Scientific) and $40 \mu \mathrm{g}$ of protein was loaded for each sample. Proteins were separated on an SDS-polyacrylamide gel and transferred to a polyvinylidene difluoride membrane. The membranes were blocked in 5\% non-fat dry milk for $1 \mathrm{~h}$ and probed with antibodies against S100P, matrix metalloproteinase MMP1, MMP2, MMP3, MMP9, MMP11, MMP13 and MMP14 and $\beta$-actin separately at $4^{\circ} \mathrm{C}$ overnight. After incubation with peroxidase-linked secondary antibodies, immunoreactive proteins were visualized by ECL reagent (Applygen Technology, Inc., Beijing, China).

Wound healing assay. Cell migration was assessed by wound healing assay. Briefly, $\sim 5 \times 10^{5}$ cells were cultured as confluent monolayer, and wounded by scratching across the well with a $200 \mu \mathrm{l}$ pipette tip. Then, the dishes were washed by D-Hanks to remove the deciduous cells. Wounded monolayer was photographed with a $x 10$ objective lens at 0 and $24 \mathrm{~h}$ after wounding. Wound healing was quantified by measurement of the average linear speed of movement of the wound edges at the indicated time points.

Transwell invasion assay. Cell invasion assays were performed using Transwell chambers with a polycarbonate membrane (Millipore, Bedford, MA, USA) coated with $40 \mu \mathrm{l}$ diluted matrix gel (BD Biosciences, San Jose, CA, USA). Cells were trypsinized and seeded at $1 \times 10^{5}$ cells/well $/ 0.1 \mathrm{ml}$ serumfree RPMI-1640 medium in the upper chambers; $0.5 \mathrm{ml}$ of RPMI-1640 medium supplemented with 20\% FBS was added into each lower chamber. At $24 \mathrm{~h}$ after incubation, cells on the surface of the membrane were wiped off, and the membranes were fixed with $95 \%$ ethanol and stained with $1 \%$ crystal violet (Sigma-Aldrich). The invaded cells clinging to the bottom of the membrane were photographed by light microscopy at $x 20$ magnification (Olympus, Tokyo, Japan). The invaded cells were calculated for the evaluation of invasion ability in each cell line. All experiments were performed in triplicate and similar results were obtained from three independent experiments.

Chromosome analysis. To investigate why the characteristics of SACC-83-derived transfected cell lines were not in 
A

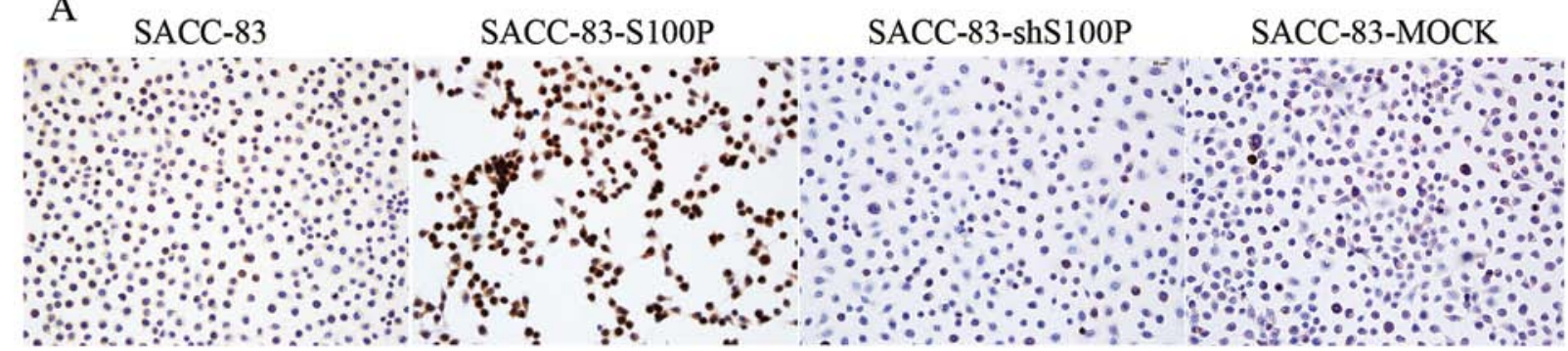

B

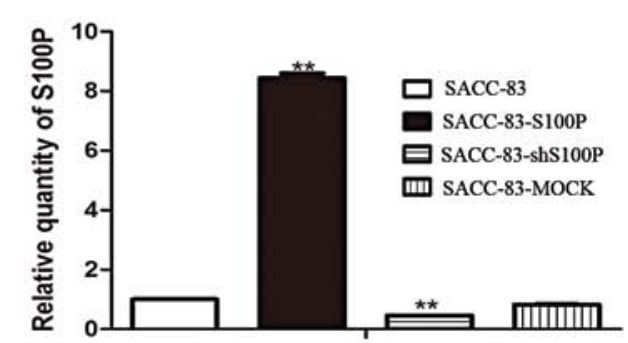

C

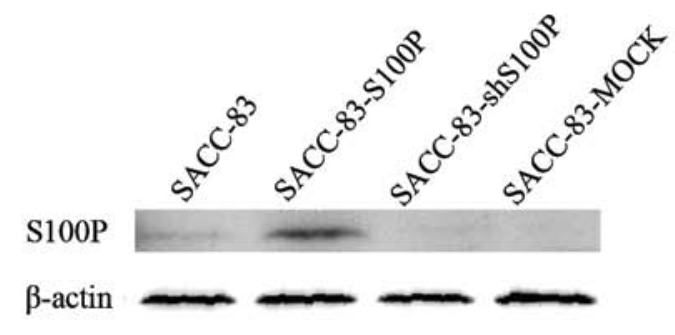

Figure 1. The identification of SACC-83-derived stably transfected cell lines with altered S100P expression levels through (A) immunostaining, (B) real-time PCR and (C) western blot assays. Amongst these cell lines, the SACC-83-S100P cell line expressed the highest level of S100P. SACC-83-shS100P cell line expressed the lowest S100P level. The expression of S100P in SACC-83-MOCK cells was somewhat lower than the parental cell line SACC-83, and a little bit higher than SACC-83-shS100P at mRNA level, but no significant difference was found amongst these three cell lines. ${ }^{* *} \mathrm{P}<0.01$.

line with S100P expression levels, chromosome analysis or karyotyping was performed to evaluate the number and structure of the chromosomes. Briefly, SACC-83 cells were cultured in nutrient-enriched media to promote cell division. The chromosomes were then isolated from the nucleus of the cells and placed on a slide for chromosome analysis. The abnormalities of the chromosome were evaluated by a genetics specialist.

Statistical analysis. The data are expressed as the means \pm SD and compared by the one-way ANOVA test through SPSS software, version 13.0 (SPSS, Inc., Chicago, IL, USA). P<0.05 was considered to indicate a statistically significant difference.

\section{Results}

Establishment of $\mathrm{S100P}$ overexpression or knockdown SACC-83 cell lines. We successfully established an S100P overexpression cell line (SACC-83-S100P), an S100P knockdown cell line (SACC-83-shS100P) and a transfected empty vector cell line (SACC-83-MOCK) using G418 selection and single cell cloning method. The cell lines were identified by immunostaining, real-time PCR and western blot assays. Immunostaining results showed that S100P protein was highly expressed in SACC-83-S100P, low expressed in SACC-83-shS100P and had a similar expression level with the parental cell line SACC-83 (Fig. 1A). Real-time PCR results showed that the mRNA level of S100P was $1.00 \pm 0.001$ in SACC-83, 8.44 \pm 0.16 in SACC-83-S100P, 0.46 \pm 0.001 in SACC-83-shS100P. Compared with SACC-83, SACC-83S100P cells produced $>8$-fold higher levels of S100P mRNA; SACC-83-shS100P cells yielded 54\% lower levels of S100P mRNA, while SACC-83-MOCK cells expressed comparable levels of S100P (Fig. 1B). Western blot results showed that
S100P protein expression pattern was similar as its mRNA expression pattern. SACC-83 expressed a very faint band; SACC-S100P expressed the highest level of S100P protein amongst the four cell lines; and S100P protein was almost undetectable in SACC-83-shS100P and SACC-83-MOCK cell lines (Fig. 1C).

Migration and invasion ability of the SACC-83-derived cell lines. After identification of S100P status in the stably transfected SACC-83 cell lines, we next examined their biological behavior. As S100P was reported to be related to cancer metastasis, we examined their migration and invasion ability. Notably, both overexpression and knockdown of S100P could stimulate the cell migration and invasion. The migration speeds of SACC-83, SACC-83-S100P, SACC-83-shS100P and SACC-83-MOCK were $11 \pm 1.56,14.81 \pm 0.25,22.93 \pm 2.17$ and $19.71 \pm 1.58 \mu \mathrm{m} / \mathrm{h}$, respectively (Fig. 2). The relative invaded tumor cells of SACC-83, SACC-83-S100P, SACC-83-shS100P and SACC-83-MOCK in Transwell assay were $1 \pm 0.30$, $2.14 \pm 0.30,2.32 \pm 0.27$ and $2.92 \pm 0.32$ (Fig. 3). The migration and invasion abilities were not related to S100P expression in these cells. SACC-83-shS100P and SACC-83-MOCK with lower levels of S100P had higher migration and invasion ability compared to SACC-83-S100P. Moreover, SACC-83S100P, SACC-83-shS100P and SACC-83-MOCK had higher migration and invasion ability compared with the parental cell line SACC-83. The status of S100P was neglected in these stably transfected SACC-83 derived cell lines.

Exogenous DNA incorporation into genome alters the metastasis-related gene expression pattern. To understand why these SACC-83-derived cell lines represented the undesirable biological abilities, we performed western blot analysis to detect the changes of MMP family proteins that were reported 


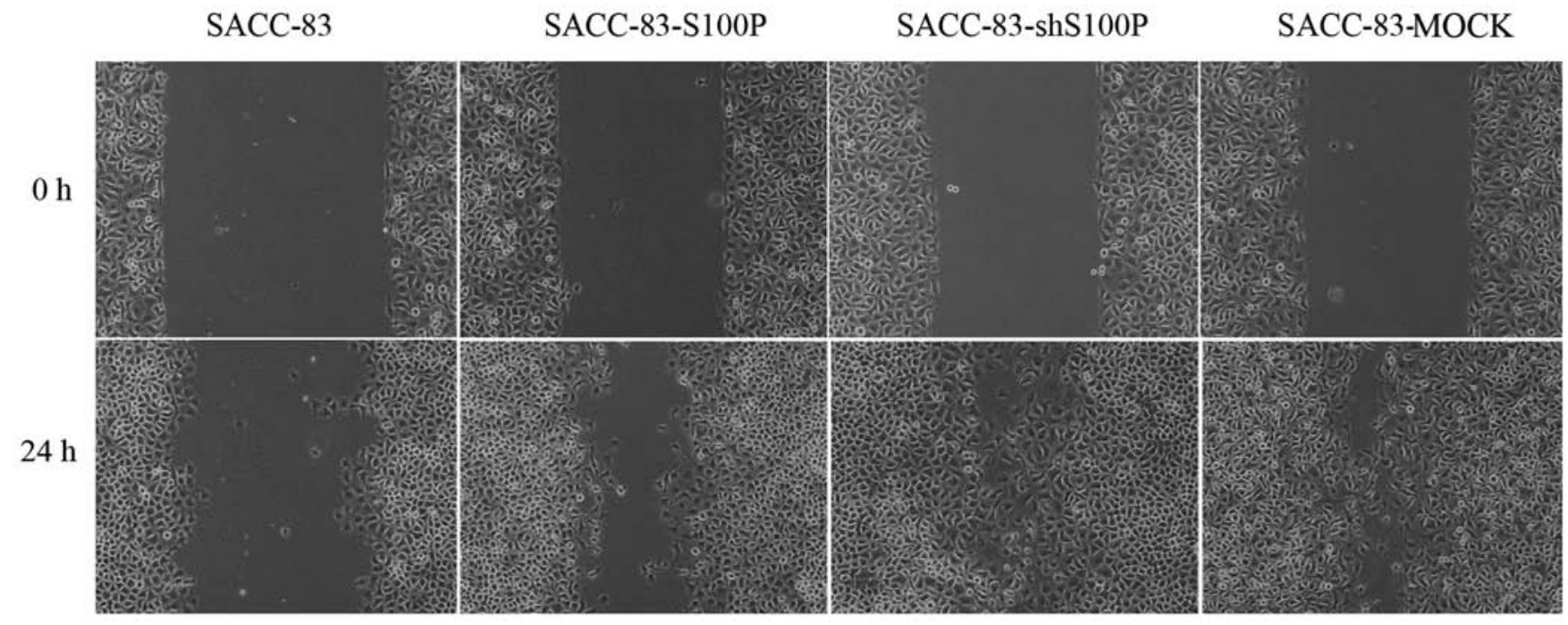

Figure 2. Migration ability of the four cell lines was observed by wound healing assay and the measuring point was 0 and $24 \mathrm{~h}$ after wounding. Original magnification, $\mathrm{x} 10$. The average migration speed $(\mu \mathrm{m} / \mathrm{h})$ was calculated by migration distance (bilateral) divided by 24 . The SACC-83-shS100P and SACC-83-MOCK exhibited stronger migration ability.

\section{SACC-83}

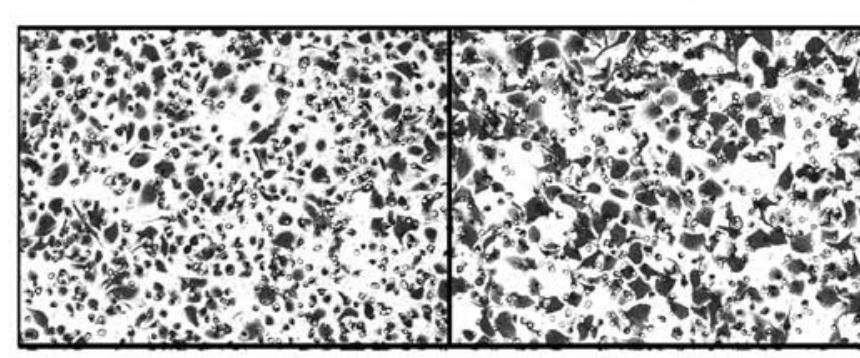

Figure 3. The invasion ability of the four cell lines was measured using the Transwell invasion assay. Original magnification, $\mathrm{x} 20$. The invaded cells were shown on the bottom surface of the membrane $24 \mathrm{~h}$ post-inoculation. The results showed that SACC-83-shS100P and SACC-83-MOCK demonstrated stronger invasion ability.

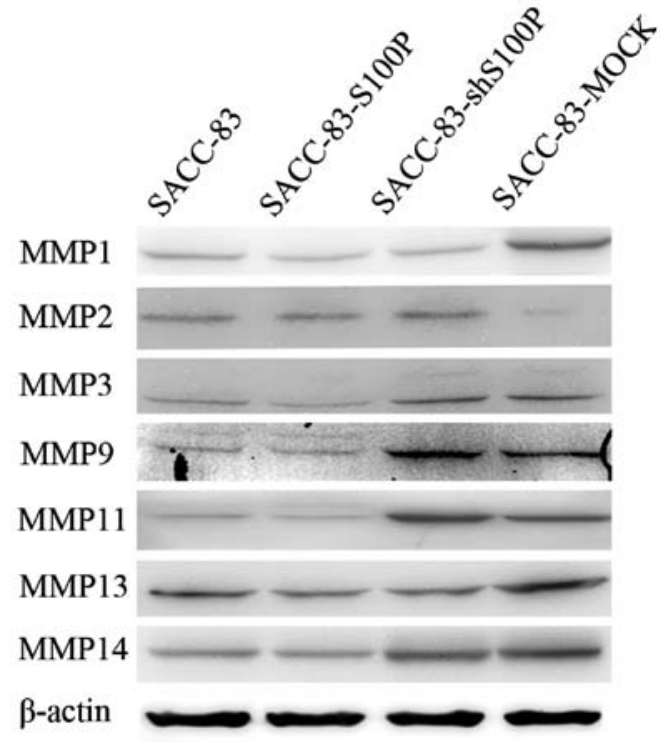

Figure 4. The differential expression genes at the protein level in SACC-83 and its derived cell lines by western blot analysis. The SACC-83-shS100P cell line expressed a higher level of MMP3, MMP9, MMP11 and MMP14 than SACC-83. The SACC-83-MOCK cell line expressed a higher level of MMP1, MMP9, MMP11 and MMP14 and a lower level of MMP2 than SACC-83. to be required in the process of the migration and invasion of SACC. As indicated in Fig. 4, the SACC-83-S100P cell line expressed the highest MMP2 compared to the other cell lines. The expression of MMP3, MMP9, MMP11 and MMP14 in the SACC-83-shS100P cell line was higher than in SACC-83. Compared to the parental cell line SACC-83, the SACC-83MOCK cell line expressed higher level of MMP1, MMP9, MMP11 and MMP14 and lower level of MMP2. The results indicated that different molecular changes caused by the random incorporation of exogenous DNA into chromosome led to the same consequence, that is, the required migration and invasion abilities were greater in the stably transfected cell lines than in SACC-83.

Chromosome analysis. To investigate why random incorporation of exogenous DNA into genome generated unexpected results, we performed chromosome analysis. The results showed that SACC-83 contained hypotetraploid karyotyping with $88 \pm 2$ chromosomes. Most chromosomes presented the abnormal morphology with high variance (Fig. 5). This result indicated that we should be cautious when editing a genome in cancer cells with high variance. 


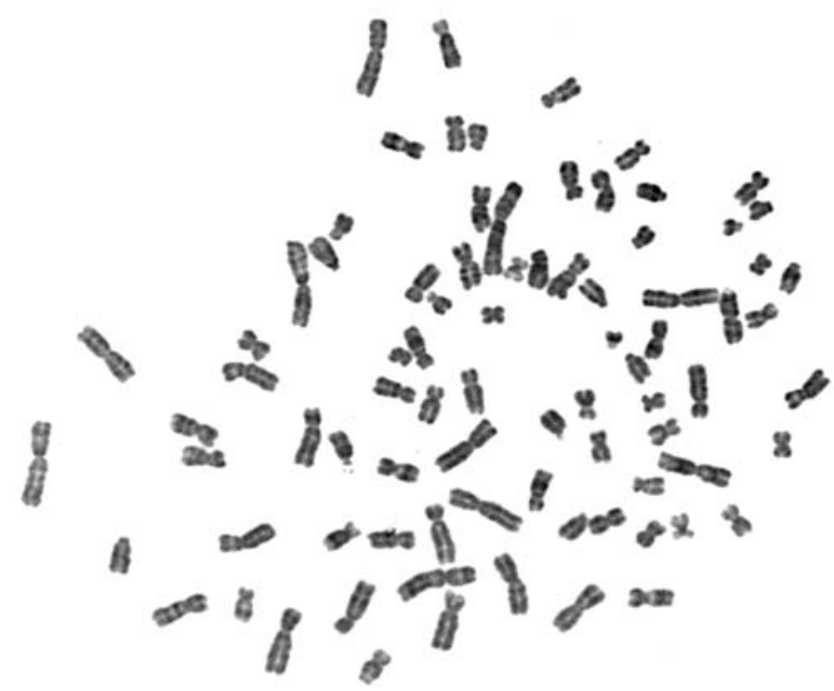

Figure 5. Chromosome analysis of SACC-83 cells. The genome of SACC-83 presents hypotetraploid karyotyping with high variance.

\section{Discussion}

Cancer biology is a major part of the life sciences, and transfection is very useful technology to investigate the gene's function in a cancer cell. In general, we only pick up the desired stably-transfected cell lines to perform the experiments and explain the expected outcomes. Everything is investigated under the pre-set conditions within our knowledge, however, something may be important, but neglected owing to this orientation. In order to avoid drawing any biased conclusion derived from the stably-transfected cancer cell lines, we performed the present study using stably S100P overexpressed and knocked down SACC-83 cells as our example to demonstrate the effect of exogenous DNA stress on the required function of the transformed cells already containing high genome variance. The reason why we selected this gene is that $\mathrm{S} 100 \mathrm{P}$ is found to be differentially expressed in the homologous but different lung metastatic ability cell lines, SACC-83 and SACC-LM, in our previous study (9). S100P has been reported to serve many important functions in diverse origins of cancer, such as stimulation proliferation, survival, motility and invasiveness (10-13).

After we established and identified the stable cell lines, we first analyzed their biological behaviors, and found that S100P expression level did not govern the mobility and invasion abilities of the stably transfected SACC-83 cell lines; both overexpressed and knocked down S100P cell lines had higher mobility and invasion abilities than the parental cell line SACC-83. Moreover, the mock-transfected cell line SACC-83-MOCK had the greatest invasion abilities amongst SACC-83-S100P, SACC-83-shS100P, SACC-83-MOCK and SACC-83 cell lines. The knockdown cell line SACC83 -shS100P had the highest mortality ability. The convoluted results led us to investigate the underlying mechanism. It is well known that cell behavior is influenced by the interaction of molecules within the cell, and MMPs play a crucial role in the extracellular matrix breakdown process of cancer metastasis (14). MMPs are a family of enzymes with the ability to degrade all types of extracellular matrix. Previous studies showed that the function of S100P was associated with the MMP family (15). Thus, we investigated some typical molecules in MMP families. Results analysis showed that different MMP molecules exhibited different expression abundance in these SACC cell lines, such as MMP1 in SACC-83-MOCK, MMP2 in SACC-83-S100P and MMP9 in SACC-83-shS100P were highly expressed, respectively. Moreover, the biological behaviors of the four SACC cell lines were supported by the expression pattern of MMPs but not by those of S100P. However, their invasion and migration abilities were confirmed to be caused by different molecular mechanism, which may be due to the exogenous DNA random incorporation.

Next, we examined the karyotyping of the parental cell line SACC-83 and tried to find the explanation. The result showed that the genome of SACC-83 had high genetic variance. We speculated that a cancer cell with high genetic variance plus the exogenous DNA random incorporation transfection may cause the gene expression pattern change in the present study, which was supported by previous studies $(16,17)$. Thus, although the stably transfected SACC cell lines exhibited the high or low expression of target gene S100P, the motility and invasiveness of the four SACC cells were not governed by S100P expression level, and were not consistent with the previous reports on S100P function during cancer metastasis (18). The random insertion of exogenous gene in a cell with high genetic variance may be the major reason for this unexpected phenomenon. Studies regarding stable transfection exogenous DNA into a cancer cell should consider the status of the genetic variance in the targeted cancer cells in order to avoid any potential bias and unreliable conclusions derived from the stably transfected cancer lines.

In addition, given that virus infection is a common inducer for certain types of cancer characteristics of the tumor cells, it may be a result of an exogenous random insertion to the chromosome, thus leading to genetic variance and the shift in gene expression profile of cancer cells.

In conclusion, although the establishment of stably transfected cancer cell lines is a common method to investigate the function of a target gene, the status of genetic variance in a cancer cell should be considered in order to avoid any potential unreliable conclusion.

\section{Acknowledgements}

The present study was supported by a Research Grant from the National Nature Science Foundation of China (grant no. 81271150).

\section{References}

1. Stuchbury G and Munch G: Optimizing the generation of stable neuronal cell lines via pre-transfection restriction enzyme digestion of plasmid DNA. Cytotechnology 62: 189-194, 2010.

2. Glover DJ, Lipps HJ and Jans DA: Towards safe, non-viral therapeutic gene expression in humans. Nat Rev Genet 6: 299-310, 2005.

3. Kim TK and Eberwine JH: Mammalian cell transfection: the present and the future. Anal Bioanal Chem 397: 3173-3178, 2010.

4. Kakudo T, Chaki S, Futaki S, et al: Transferrin-modified liposomes equipped with a $\mathrm{pH}$-sensitive fusogenic peptide: an artificial viral-like delivery system. Biochemistry 43: 5618-5628, 2004. 
5. Kursa M, Walker GF, Roessler V, et al: Novel shielded transferrin-polyethylene glycol-polyethylenimine/DNA complexes for systemic tumor-targeted gene transfer. Bioconjug Chem 14: 222-231, 2003

6. Zanta MA, Belguise-Valladier P and Behr JP: Gene delivery: a single nuclear localization signal peptide is sufficient to carry DNA to the cell nucleus. Proc Natl Acad Sci USA 96: 91-96, 1999.

7. Barrett LE, Sul JY, Takano H, et al: Region-directed phototransfection reveals the functional significance of a dendritically synthesized transcription factor. Nat Methods 3: 455-460, 2006.

8. Negrini S, Gorgoulis VG and Halazonetis TD: Genomic instability - an evolving hallmark of cancer. Nat Rev Mol Cell Biol 11: 220-228, 2010.

9. Wang L, Wang Y, Bian $\mathrm{H}$, et al: Molecular characteristics of homologous salivary adenoid cystic carcinoma cell lines with different lung metastasis ability. Oncol Rep 30: 207-212, 2013.

10. Cheng YS, Jordan L, Rees T, et al: Levels of potential oral cancer salivary mRNA biomarkers in oral cancer patients in remission and oral lichen planus patients. Clin Oral Investig: Jul 28, 2013 (Epub ahead of print).

11. Arumugam T, Simeone DM, Van Golen K, et al: S100P promotes pancreatic cancer growth, survival, and invasion. Clin Cancer Res 11: 5356-5364, 2005.
12. Ge F, Wang $\mathrm{C}$, Wang $\mathrm{W}$, et al: $\mathrm{S} 100 \mathrm{P}$ predicts prognosis and drug resistance in gastric cancer. Int J Biol Markers 28: e387-e392, 2013.

13. Yuan RH, Chang KT, Chen YL, et al: S100P expression is a novel prognostic factor in hepatocellular carcinoma and predicts survival in patients with high tumor stage or early recurrent tumors. PLoS One 8: e65501, 2013.

14. Deryugina EI and Quigley JP: Matrix metalloproteinases and tumor metastasis. Cancer Metastasis Rev 25: 9-34, 2006.

15. Namba T, Homan T, Nishimura T, et al: Up-regulation of S100P expression by non-steroidal anti-inflammatory drugs and its role in anti-tumorigenic effects. J Biol Chem 284: 4158-4167, 2009.

16. Denko NC, Giaccia AJ, Stringer JR, et al: The human Ha-ras oncogene induces genomic instability in murine fibroblasts within one cell cycle. Proc Natl Acad Sci USA 91: 5124-5128, 1994.

17. Gorgoulis VG, Vassiliou LV, Karakaidos P, et al: Activation of the DNA damage checkpoint and genomic instability in human precancerous lesions. Nature 434: 907-913, 2005.

18. Barry S, Chelala C, Lines K, et al: S100P is a metastasis-associated gene that facilitates transendothelial migration of pancreatic cancer cells. Clin Exp Metastasis 30: 251-264, 2013. 